\title{
Determining the Ethanol Concentrations in Ethyl Alcohol-Based Hand Sanitizers with Different Expiration Dates Using Fourier-Transform Infrared Spectroscopy (FT-IR)
}

\author{
Max Shlafstein ${ }^{\dagger 1}$, Emily Hannah ${ }^{\dagger 1}$, and Ling Hao*1 \\ ${ }^{1}$ Department of Chemistry, The George Washington University, $80022^{\text {nd }}$ St. NW, Washington, DC 20052, USA.
}

\begin{abstract}
Hand sanitizer has proved to be an essential public health tool in slowing the spread of the coronavirus (COVID-19). However, amid the COVID-19 pandemic, the availability of recently manufactured hand sanitizer can be limited. The product expiration date set by manufacturers is also minimally regulated. The efficacy of hand sanitizer is entirely determined by its most volatile ingredient, alcohol, which must remain at a concentration greater than $60 \%$ (v/v) to kill bacterial and viral pathogens. In our undergraduate Chem 2123W course (Introductory Quantitative Analysis Laboratory) in spring 2021, we designed and conducted an experiment to investigate the alcohol concentration of ethyl alcohol-based commercial hand sanitizers with varying expiration dates. The presence of a primary alcohol functional group in ethyl alcohol can be observed by Fourier-transform infrared spectroscopy (FTIR). Through a series of standard additions of ethyl alcohol to the diluted hand sanitizer sample, we measured the changes in the integration of the FT-IR primary alcohol C-O stretch. The signal changes in integration were used to generate a standard addition curve using ethanol concentration and calculate the initial ethanol concentration of each sample. Our approach accounts for this matrix effect and does not rely on a compositional approximation. All the samples studied were found to exceed the minimum concentration required to effectively exhibit antimicrobial properties despite three of the four samples being expired. However, the expired samples remained close to the $60 \%$ threshold and with consideration of error analysis, the effectiveness of these hand sanitizers remains uncertain. The samples analyzed in this study varied in reported initial ethyl alcohol concentration, manufacturer, color, perfume, and other ingredients. The heterogeneity of our samples coupled with our consistent results provided insight into consumer use of expired ethyl alcohol-based hand sanitizers.
\end{abstract}

Keywords: Hand Sanitizer, COVID-19, Pandemic, Ethanol, FT-IR, Standard Addition, Expiration dates

\section{INTRODUCTION}

The recent coronavirus (COVID-19) pandemic fundamentally altered the role of public health in mass consumer's everyday lives. Many public health campaigns were promoting the effectiveness of maintaining hand hygiene during COVID-19, with strategies including hand washing and alcohol-based hand rubs (ABHR) ${ }^{1,2}$ These efforts were aimed to reduce the spread of the virus which is transmitted through mucous membranes of the nose, eyes, and mouth. ${ }^{3}$ While effective, during the peak of the COVID-19 pandemic the supply of hand sanitizer products could simply not meet the incredibly high demand. This forced consumers to search their homes for hand sanitizer products purchased years prior. As demand for hand sanitizer surges, so has the emergence of substandard products. ${ }^{4}$ Excessive hoarding and price gouging have also exacerbated the scarcity of these personal hygiene products. ${ }^{5}$ These issues built the foundation for our "do it yourself" (DIY) experiment in our undergraduate Chem 2123W course: Introductory Quantitative Analysis Laboratory. The shift to online coursework provided flexibility in experimental design and allowed for investigation into these commercial hand sanitizer products.
The product expiration date set by manufacturers is minimally regulated and a fairly inexact science. ${ }^{6}$ In fact, most of the weight placed in product expiration dates is rooted in consumer perception of "peak quality" of a particular good. ${ }^{6} \mathrm{We}$ see this often in the food industry where date-labeling contributes to a significant amount of avoidable food waste. ${ }^{6,7}$ Consumers heavily weigh the expiration date which is based on ill-defined guidelines, and food waste amasses. ${ }^{6}$ In a public health crisis where there is a shortage and/or limited access (due to cost, supply, etc.) to food, goods, and services, placing less weight on these arbitrary recommendations and educating consumers that this date is more suggestive is certainly plausible for conservation and resourcefulness of goods. ${ }^{1}$

We aim to test the how different expiration dates influence the alcohol concentration in hand sanitizer. We propose that a recently expired hand sanitizer may be included as a potential alternative when resources are not available in extenuating circumstances in public health emergency. This may further discourage consumers from pursuing an alternative such as making their own alcohol-based hand sanitizers at home which poses additional health and safety risks as outlined by the FDA. $^{8}$ 


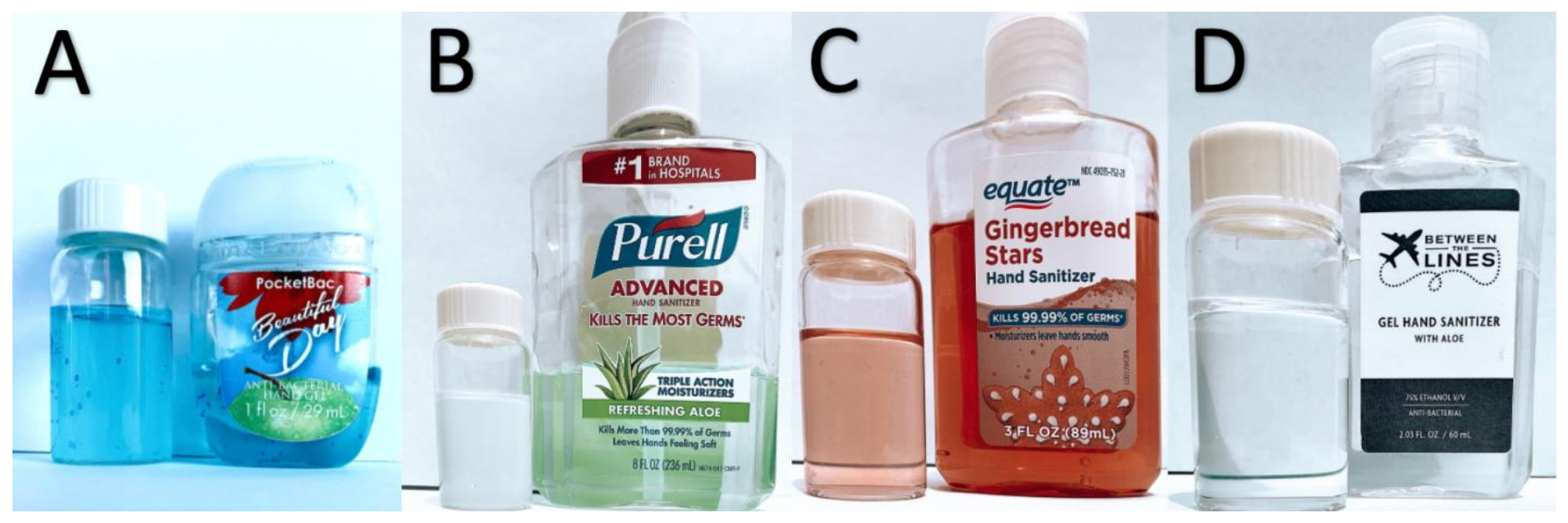

Figure 1: Hand sanitizer samples and $20 \mathrm{~mL}$ diluted hand sanitizers in scintillation vials. Sample A: PocketBac Beautiful Day expiration: July 2018. Sample B: Purell Advanced Hand Sanitizer, Refreshing Aloe - expiration: August 2019. Sample C: Equate Gingerbread Stars Hand Sanitizer - expiration: October 2020. Sample D: Between the Lines Gel Hand Sanitizer with Aloe expiration June 2022.

Efficacy of Alcohol-Based Hand Sanitizers. The active ingredient in all alcohol-based hand sanitizers is ethyl alcohol or isopropyl alcohol, at a concentration of $60-95 \%(\mathrm{v} / \mathrm{v})$. This concentration of ethanol has been tested and proved effective against common bacteria including, Serratia marcescens, Pseudomonas aeruginosa, Escherichia coli, Salmonella typhosa, Staphylococcus aureus, and Streptococcus pyogenes. ${ }^{9,10}$ In the presence of ethanol, the hydrophobic forces holding the lipid cell membrane intact are weakened as ethanol is less polar than water, causing an increase in membrane permeability and leakage of life sustaining intracellular components. ${ }^{11,12}$ It is important to note that $60 \%$ alcohol is the lowest concentration that remains effective against these common bacteria; however $100 \%$ alcohol was shown to be ineffective against Staphylococcus aureus and Streptococcus pyogenes as the high concentration of alcohol will cause instant coagulation of the peptidoglycan layer which protects the bacteria from further damage. ${ }^{9,13}$ In relation to viral pathogens, common hand sanitizer at $60-80 \%$ ethanol has been shown to be effective against the protein capsid of enveloped viruses such as the coronavirus; however, higher concentrations of $\sim 95 \%$ are needed for efficacy against non-enveloped viruses such as poliovirus and adenovirus. ${ }^{14}$ While hand sanitizer is effective in vitro, practical application studies show the true value of alcohol-based hand rub (ABHR). A three-year study of an extended care facility by Fendler et al. found an average decrease in the bacterial and viral infection rate of $30.4 \%$ for patients on the floor where hand sanitizer was distributed and dispensers were available in common areas in comparison to the floor without these interventions. ${ }^{15}$

Rationale and Methods to Quantify Ethanol. Ethanol has a boiling point of $78.2{ }^{\circ} \mathrm{C}$, which is relatively volatile in comparison to the boiling point of water, $100{ }^{\circ} \mathrm{C} .{ }^{16,17}$ As ethanol is the active ingredient in hand sanitizers, it will evaporate over extended periods of time leaving the solution with a higher percentage of water rendering its antimicrobial properties ineffective. Through extensive research, the World Health
Organization (WHO) has recommended a $80 \%$ (v/v) ethanol, $1.45 \%(\mathrm{v} / \mathrm{v})$ glycerol, and $0.125 \%(\mathrm{v} / \mathrm{v})$ hydrogen peroxide solution as an effective and versatile hand sanitizer solution. ${ }^{18}$ However, many commercial hand sanitizers are manufactured as $60-62 \%(\mathrm{v} / \mathrm{v})$ ethanol, already dangerously close to the efficacy threshold. ${ }^{19}$ Due to the decline of hand sanitizer availability during the COVID-19 pandemic, the use of expired hand sanitizer has likely greatly increased.

Many techniques have been used to quantify ethanol concentration including basic redox titrations, ${ }^{20}$ electroanalytical flow injection analysis, ${ }^{21}$ amperometric biosensing, ${ }^{22}$ and gas chromatography coupled mass spectrometry. ${ }^{23}$ While effective, these techniques are often time consuming, require extensive training, and are destructive to the sample. ${ }^{24}$ Fourier-transform infrared spectroscopy (FT-IR) was chosen for this study for its ease of use, nondestructive nature, and high accuracy. In determining the concentration of ethanol from water, the main components of hand sanitizer, it is important to consider the similarities of the two molecules. Both display hydrogen bonding and have distinctive hydroxyl groups, however, ethanol has a carbon-oxygen bond which can be identified using FT-IR and is shown at $1044-1045 \mathrm{~cm}^{-1}$ on the transmittance spectrum. ${ }^{25}$ This methodology of using the C-O bond has been used by FT-IR manufacturers PerkinElmer and Shimadzu to determine the effectiveness of FT-IR estimation of ethanol percentage in prepared solutions of hand sanitizer. ${ }^{26,27}$ However, both of these studies use a calibration curve technique, creating samples of increasing ethanol concentration based on the WHO guidelines for hand sanitizer and using this curve as a reference with water as the blank. This approach may produce a matrix effect in which the analytical signal is altered due to a component of the hand sanitizer that is not the analyte.

In this study, a standard addition curve is created by adding known quantities of the ethanol to the unknown hand sanitizer solution, to create a linear instrumental response, which is used to determine the original concentration of ethanol. Our approach accounts for this matrix effect and does not rely 
on a compositional approximation. While the accuracy of measurements is improved by using a standard addition curve, there are still limitations in this study. The limited availably of expired hand sanitizers and diversity of brands and bottle designs introduce confounding variables besides time that could affect the ethanol concentration. The goal of this study is to investigate the concentration of alcohol in a range of hand sanitizers with different expiration dates to determine their efficacy past that date. As little research has been done to quantify the efficacy of hand sanitizer past the expiration date, we aim to provide a quantitative analysis of the ethanol concentration in these products.

\section{EXPERIMENTAL PROCEDURES}

Materials. Pure ethyl alcohol was obtained from Sigma-Aldrich (459844-4L, Lot \#SHBL6038; 200 proof, ACS reagent, $\geq$ $99.5 \%$ ). Deionized water was obtained from The George Washington University Science and Engineering Hall laboratory deionized water line. Four commercial hand-sanitizer samples (Figure 1) with varying expiry dates were procured for the experimental standard additions: (A) PocketBac Beautiful Day (1 fl. oz., a turquoise transparent gel with approximately $1 \mathrm{~mm}$ solid blue beads mixed homogeneously throughout), (B) Purell Advanced Hand Sanitizer, Refreshing Aloe (8 fl. oz., a green/clear transparent gel), (C) Equate Gingerbread Stars Hand Sanitizer (3 fl. oz., an orange transparent gel), (D) Between the Lines Gel Hand Sanitizer with Aloe (2.03 fl. oz., a colorless gel).

The following procedure was performed for Samples A-D (Figure 2). The scintillation vials and pure ethyl alcohol were capped for the duration of the experiment (except during standard additions or transfers) to minimize evaporation.

Sample Preparation and Standard Addition. $5 \mathrm{~mL}$ of the sample was transferred to a $20 \mathrm{~mL}$ scintillation vial with a $1 \mathrm{~mL}$ syringe. The sample was diluted with $8 \mathrm{~mL}$ of deionized water. The scintillation vial was capped and gently mixed. The initial sample of diluted hand sanitizer was noted as "X1" where $\mathrm{X}$ is the letter of the corresponding sample. $1 \mathrm{~mL}$ successive additions of pure ethyl alcohol were added to the scintillation vial. The vial was capped and gently mixed after each standard addition. A spectrum of the standard addition was generated. Each successive standard addition to the initial sample was labeled "X2-X5." A total of four standard additions and five spectra per sample were completed.

The sample preparation and standard additions were both completed with a $1 \mathrm{~mL}$ syringe with an instrumental error of $0.05 .{ }^{28}$ The propagated error for each $1 \mathrm{~mL}$ in the sample preparation is described in Table 1. The initial volume of the diluted hand sanitizer $\left(\mathrm{V}_{0}\right)$ for Samples A-D was $13.00 \pm 0.18$ $\mathrm{mL}$ (Table 1). The volume of the standard pure ethyl alcohol $\left(\mathrm{V}_{\mathrm{s}}\right)$ and associated propagated error for each $1 \mathrm{~mL}$ standard addition is also noted in Table 1 . The initial concentration of the aliquot of pure ethyl alcohol standard $\left([\mathrm{S}]_{\mathrm{i}}\right)$ was 200 proof, or
$100 \%$ pure by volume with an error of $0.5 \%$ (ACS reagent, $\geq$ 99.5\%). (V) represents the volume of the diluted hand sanitizer plus the standard addition (Table 1). The reported initial concentration of ethyl alcohol in each sample and expiration dates were located on the container of each bottle.

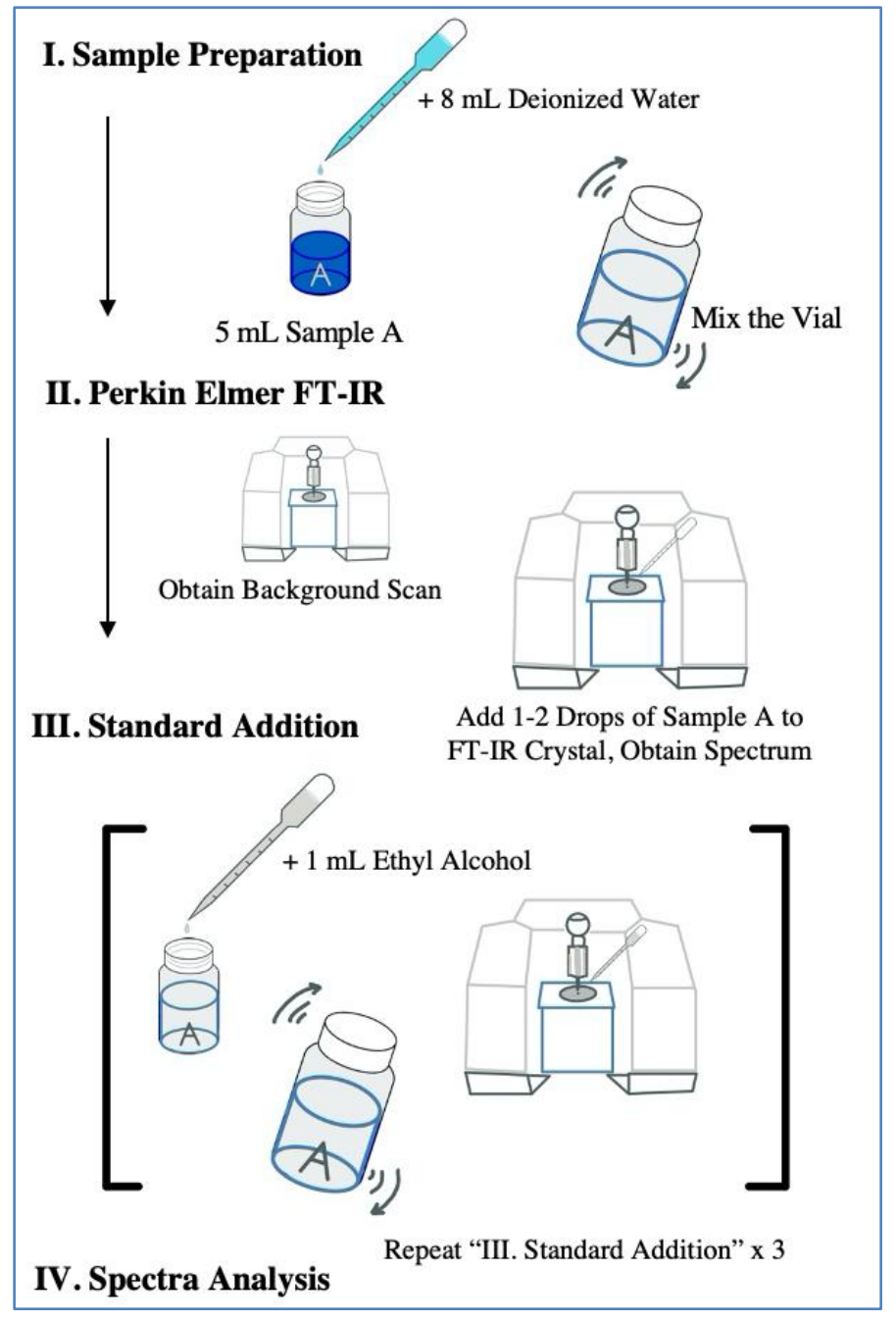

Figure 2: Experimental Workflow I. Sample Preparation: 5 $\mathrm{mL}$ of Sample A was transferred to a $20 \mathrm{~mL}$ scintillation vial. 8 $\mathrm{mL}$ of deionized water was added to the scintillation vial using a pipette and the vial was mixed. II. Perkin-Elmer FT-IR: Background scan was obtained. 1-2 drops of Sample A were added onto FT-IR crystal using a pipette. FT-IR Spectrum of diluted Sample A was obtained. III. Standard Addition: $1 \mathrm{~mL}$ successive additions of pure ethyl alcohol were added to Sample A using a pipette. The vial was mixed. FT-IR Spectrum of Sample A was obtained. Three more standard additions were completed. IV. Spectra Analysis of FT-IR was completed. 
Table 1: Standard Additions of Pure Ethyl Alcohol to a Single Solution of Hand Sanitizer (Samples A-D)

\begin{tabular}{|c|c|c|c|c|c|c|c|c|c|c|}
\hline Name & Expiration & $\begin{array}{c}\text { Reported EtOH } \\
(\%)\end{array}$ & Sample & $V_{0}(m L)$ & $\begin{array}{c}\text { Theoretical EtOH } \\
\text { in } V_{0}(\mathrm{~mL})\end{array}$ & $\mathrm{V}_{\mathrm{s}} \operatorname{EtOH}(\mathrm{mL})$ & $V(m L)$ & $\mathbf{I}_{(\mathrm{s}+\mathbf{x})}$ & $S_{i} \times V_{s} / V_{0}$ & $\mathbf{I}_{(\mathbf{s}+\mathbf{x})} \times \mathbf{V} / \mathbf{V}_{\mathbf{0}}$ \\
\hline \multirow{5}{*}{$\begin{array}{c}\text { PocketBac Beautiful } \\
\text { Day } \\
\text { Hand Sanitizer }\end{array}$} & \multirow{5}{*}{$\begin{array}{l}\text { July - } 2018 \\
\text { (32 mo.) }\end{array}$} & \multirow{5}{*}{68} & $\mathrm{~A} 1$ & $13.00 \pm 0.18$ & \multirow{5}{*}{$3.40 \pm 0.11$} & $0.00 \pm 0.00$ & $13.00 \pm 0.18$ & $411.43 \pm 0.1$ & $0.00 \pm 0.00$ & $411 \pm 8$ \\
\hline & & & $\mathrm{A} 2$ & $13.00 \pm 0.18$ & & $1.00 \pm 0.05$ & $14.00 \pm 0.19$ & $478.63 \pm 0.1$ & $0.0769 \pm 0.004$ & $515 \pm 10$ \\
\hline & & & $\mathrm{A} 3$ & $13.00 \pm 0.18$ & & $2.00 \pm 0.07$ & $15.00 \pm 0.19$ & $542.43 \pm 0.1$ & $0.154 \pm 0.006$ & $626 \pm 12$ \\
\hline & & & A4 & $13.00 \pm 0.18$ & & $3.00 \pm 0.09$ & $16.00 \pm 0.20$ & $586.79 \pm 0.1$ & $0.231 \pm 0.007$ & $722 \pm 13$ \\
\hline & & & A5 & $13.00 \pm 0.18$ & & $4.00 \pm 0.10$ & $17.00 \pm 0.21$ & $617.15 \pm 0.1$ & $0.308 \pm 0.009$ & $807 \pm 15$ \\
\hline \multirow{5}{*}{$\begin{array}{l}\text { Purell Advanced } \\
\text { Refreshing Aloe } \\
\text { Hand Sanitizer }\end{array}$} & \multirow{5}{*}{$\begin{array}{l}\text { Aug - } 2019 \\
\text { (19 mo.) }\end{array}$} & \multirow{5}{*}{70} & B1 & $13.00 \pm 0.18$ & \multirow{5}{*}{$3.50 \pm 0.11$} & $0.00 \pm 0.00$ & $13.00 \pm 0.18$ & $410.77 \pm 0.1$ & $0.00 \pm 0.00$ & $411 \pm 8$ \\
\hline & & & B2 & $13.00 \pm 0.18$ & & $1.00 \pm 0.05$ & $14.00 \pm 0.19$ & $477.28 \pm 0.1$ & $0.0769 \pm 0.004$ & $514 \pm 10$ \\
\hline & & & B3 & $13.00 \pm 0.18$ & & $2.00 \pm 0.07$ & $15.00 \pm 0.19$ & $534.70 \pm 0.1$ & $0.154 \pm 0.006$ & $617 \pm 12$ \\
\hline & & & B4 & $13.00 \pm 0.18$ & & $3.00 \pm 0.09$ & $16.00 \pm 0.20$ & $558.36 \pm 0.1$ & $0.231 \pm 0.007$ & $687 \pm 13$ \\
\hline & & & B5 & $13.00 \pm 0.18$ & & $4.00 \pm 0.10$ & $17.00 \pm 0.21$ & $616.96 \pm 0.1$ & $0.308 \pm 0.009$ & $807 \pm 15$ \\
\hline \multirow{5}{*}{$\begin{array}{c}\text { Equate Gingerbread } \\
\text { Stars } \\
\text { Hand Sanitizer }\end{array}$} & \multirow{5}{*}{$\begin{array}{l}\text { Oct - } 2020 \\
(5 \text { mo. })\end{array}$} & \multirow{5}{*}{62} & $\mathrm{C} 1$ & $13.00 \pm 0.18$ & \multirow{5}{*}{$3.10 \pm 0.11$} & $0.00 \pm 0.00$ & $13.00 \pm 0.18$ & $356.08 \pm 0.1$ & $0.00 \pm 0.00$ & $356 \pm 7$ \\
\hline & & & $\mathrm{C} 2$ & $13.00 \pm 0.18$ & & $1.00 \pm 0.05$ & $14.00 \pm 0.19$ & $426.47 \pm 0.1$ & $0.0769 \pm 0.004$ & $459 \pm 9$ \\
\hline & & & $\mathrm{C} 3$ & $13.00 \pm 0.18$ & & $2.00 \pm 0.07$ & $15.00 \pm 0.19$ & $453.36 \pm 0.1$ & $0.154 \pm 0.006$ & $523 \pm 10$ \\
\hline & & & $\mathrm{C} 4$ & $13.00 \pm 0.18$ & & $3.00 \pm 0.09$ & $16.00 \pm 0.20$ & $532.75 \pm 0.1$ & $0.231 \pm 0.007$ & $656 \pm 12$ \\
\hline & & & $\mathrm{C} 5$ & $13.00 \pm 0.18$ & & $4.00 \pm 0.10$ & $17.00 \pm 0.21$ & $538.15 \pm 0.1$ & $0.308 \pm 0.009$ & $704 \pm 13$ \\
\hline \multirow{5}{*}{$\begin{array}{c}\text { Between the Lines } \\
\text { Gel Hand Sanitizer } \\
\text { with Aloe }\end{array}$} & \multirow{5}{*}{$\begin{array}{c}\text { Jun - } 2022 \\
(-14 \text { mo. })\end{array}$} & \multirow{5}{*}{75} & D1 & $13.00 \pm 0.18$ & \multirow{5}{*}{$3.75 \pm 0.11$} & $0.00 \pm 0.00$ & $13.00 \pm 0.18$ & $425.71 \pm 0.1$ & $0.00 \pm 0.00$ & $426 \pm 8$ \\
\hline & & & D2 & $13.00 \pm 0.18$ & & $1.00 \pm 0.05$ & $14.00 \pm 0.19$ & $490.78 \pm 0.1$ & $0.0769 \pm 0.004$ & $529 \pm 10$ \\
\hline & & & D3 & $13.00 \pm 0.18$ & & $2.00 \pm 0.07$ & $15.00 \pm 0.19$ & $538.42 \pm 0.1$ & $0.154 \pm 0.006$ & $621 \pm 12$ \\
\hline & & & D4 & $13.00 \pm 0.18$ & & $3.00 \pm 0.09$ & $16.00 \pm 0.20$ & $549.19 \pm 0.1$ & $0.231 \pm 0.007$ & $676 \pm 13$ \\
\hline & & & D5 & $13.00 \pm 0.18$ & & $4.00 \pm 0.10$ & $17.00 \pm 0.21$ & $560.21 \pm 0.1$ & $0.308 \pm 0.009$ & $732 \pm 14$ \\
\hline
\end{tabular}

FT-IR Analysis. Perkin Elmer FT-IR Spectrometer Frontier crystal was cleaned with Kimtech Kimwipes and a background scan was obtained to calibrate the spectrometer. The sample was extracted from the scintillation vial with a pipette and 1-2 drops were transferred onto the IR crystal of the spectrometer. ${ }^{27,29}$ For Sample A, drops that contained a blue bead were avoided from being placed on the IR crystal. An IR spectrum was generated of the sample, 8 scans were performed. The IR range was set to $4000-600 \mathrm{~cm}^{-1}$.

Spectra Analysis. The five spectra for the sample were saved. The "Peak Area/Height" tool was selected. The peak around $1044-1045 \mathrm{~cm}^{-1}$ for each spectrum was highlighted and the peak area was added to the peak table for further analysis. A multiple spectra report was generated as an .rtf, "summary" and "spectrum graph" were selected under "report options" and the file was saved. Under the sample folder, the parameters were then adjusted to reflect the region of interest (ROI): $\mathrm{x}$-axis: 1125-1000 $\mathrm{cm}^{-1}$, y-axis: $95-50 \%$ Transmittance (\%T). A multiple spectra report of the region of interest was generated as an .rtf, "summary," "spectrum graph," "peak table," and "peak area/height results" were selected under "report options" and the file was saved. The ROI for each successive standard addition for Samples A-D is depicted in Figure 4. Based on the analytical signal changes for each successive standard addition of ethyl alcohol to the solution, $[\mathrm{S}]_{\mathrm{i}} * \mathrm{~V}_{\mathrm{s}} / \mathrm{V}_{0}$ was plotted against $\mathrm{I}_{\mathrm{s}+\mathrm{x}} * \mathrm{~V} / \mathrm{V}_{0}$ for Samples A-D ${ }^{30}$ A line of least squares was generated for each sample using the readings obtained from the standard additions of pure ethyl alcohol ${ }^{30}$ The $\mathrm{x}$-intercept of the plot denotes the initial concentration of ethyl alcohol in each sample of hand sanitizer $\left([\mathrm{X}]_{\mathrm{i}}\right)^{30}$.

\section{RESULTS AND DISCUSSION}

This experiment aims to determine the percentage of ethanol in commercial hand sanitizers at a variety of different expiration dates, shown in Table 1. A standard addition curve coupled with FT-IR spectroscopy measurement of the carbon-oxygen bond was selected for this study because of accuracy, speed, and elimination of unwanted instrument response due to the matrix effect. A total of eight multiple spectra reports were generated per sample including one spectrum with the default FT-IR scale shown in Figure 3.

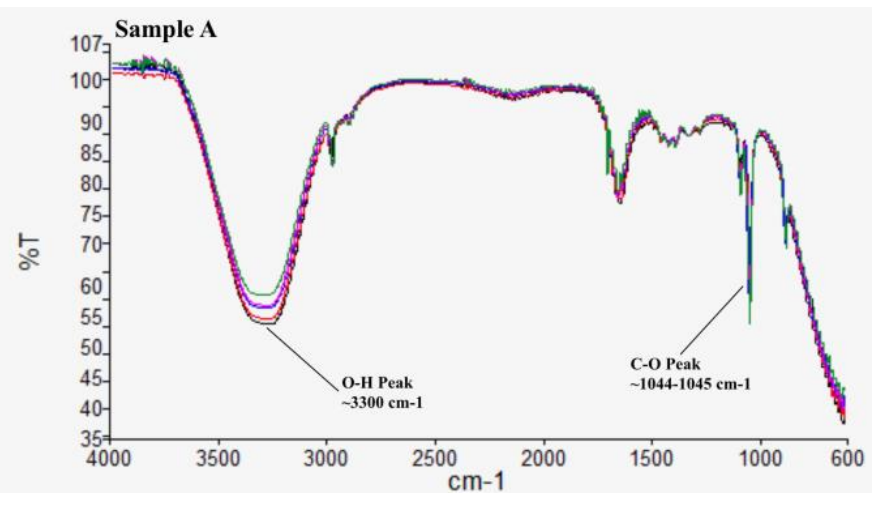

Figure 3: FT-IR (4000-600 $\left.\mathrm{cm}^{-1}\right)$ of example hand sanitizer sample after Standard Additions of Pure Ethyl Alcohol. 

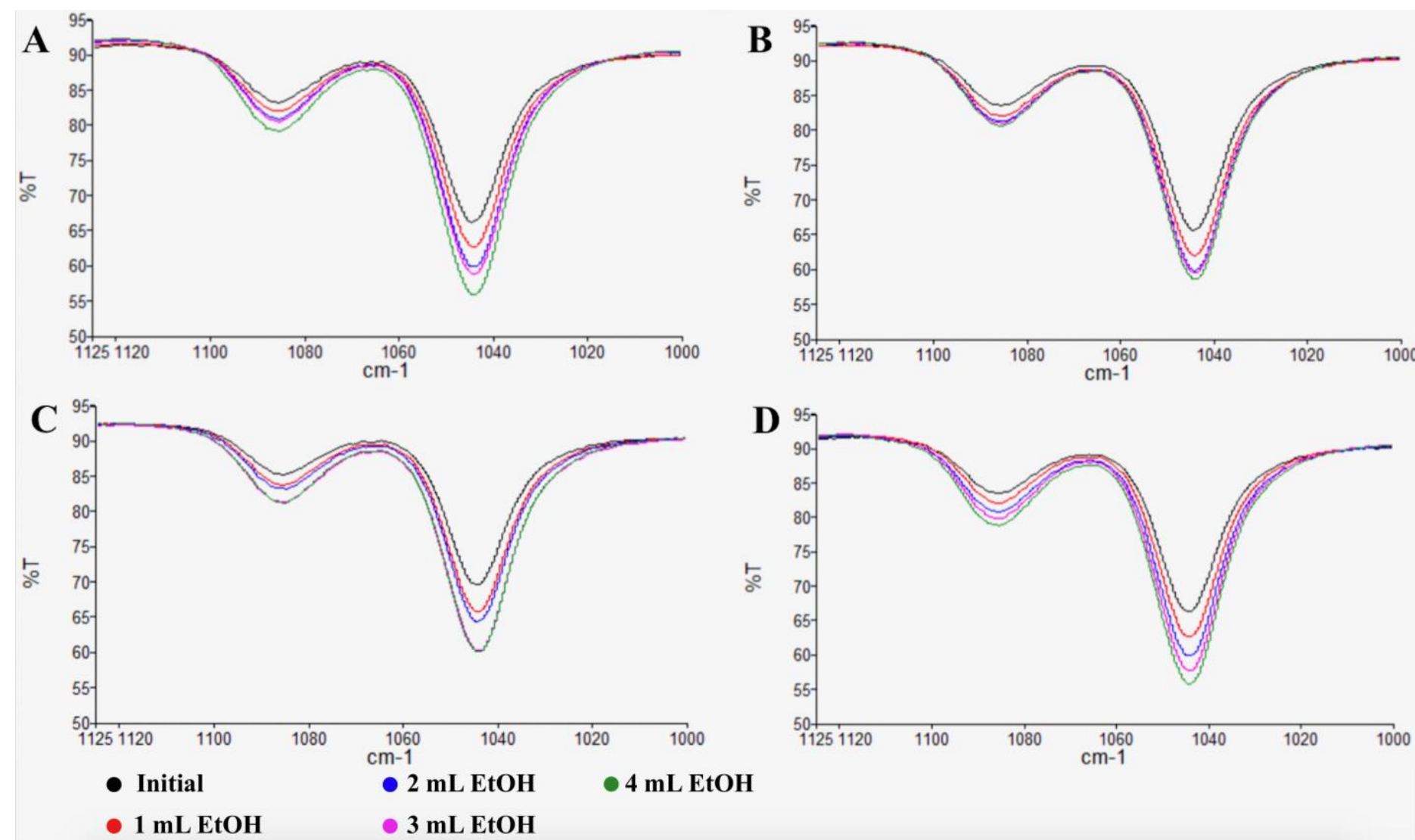

Figure 4: FT-IR Region of Interest $\left(1125-1000 \mathrm{~cm}^{-1}\right)$ for samples A-D after standard addition of Pure Ethyl Alcohol.

The FT-IR spectrum scaled to the Region of Interest (ROI) at $1125-1000 \mathrm{~cm}^{-1}$ which represents the $\mathrm{C}-\mathrm{O}$ bond of a primary alcohol is shown in Figure 4. The carbon-oxygen bond that appears at 1044-1045 $\mathrm{cm}^{-1}$ was chosen due to its distinction from other peaks and relative distance from the large oxygenhydrogen peak at $\sim 3300 \mathrm{~cm}^{-1}$. The absolute value of the peak area, or the signal of the sample and each standard addition $\left(\mathrm{I}_{\mathrm{s}+\mathrm{x}}\right)$, increased with each standard addition of pure ethyl alcohol at the ROI (Table 1, Figure 4). The error for $I_{s+x}$ was 0.1 , which is equal to the error of $\% \mathrm{~T}$ reported by the manufacterer. ${ }^{29}$ Using the Spectrum software to determine the integration at the 1044-1045 $\mathrm{cm}^{-1}$ peak, a standard addition curve could be created by comparing the ethanol concentration to the peak area and accounting for changes in volume as previously described, shown in Figure 5.

Using the standard addition calibration curve, the initial concentration of alcohol in the hand sanitizers can be calculated from the distance from the $\mathrm{x}$-intercept to the origin. The $5 \mathrm{~mL}$ sample of ABHR was initially diluted with water to decrease the viscosity of the sample. The dilution served two purposes: first, to better observe changes in ethanol concentration with each standard addition and second, to decrease the viscosity of the sample for ease of transfers and thorough mixing. This dilution was accounted for in the calculated values of ethanol in the four hand sanitizer samples shown in Table 2 using equation 1:

$$
V_{e}=\frac{\left(13 \times[X]_{i}\right)}{\left(1+[X]_{i}\right)}
$$

The Purell hand sanitizer was nineteen months past the expiration date and showed an ethanol percentage of $64.51 \%$, a loss of $0.28 \%$ per month which is still within the effective range $>60 \%$. The Equate hand sanitizer was determined as $61.77 \%$ ethanol, which is only a decrease of $0.23 \%$ over the 5 months it has expired. PocketBac hand sanitizer lost $4.8 \%$ ethanol, almost the same as the Purell, however, it expired over 32 months ago and only showed a loss of $0.15 \%$ per month to end at $63.22 \%$ ethanol. As expected, the three expired samples (A, B, and C) had a lower ethyl alcohol concentration compared to their reported initial concentration by volume.

Interestingly, the Between the Lines hand sanitizer was determined to be $80.59 \%$ ethanol, over $5 \%$ more than reported by the manufacturer. Based on these results, we propose that: 1) either manufacturer's initial concentration of ethyl alcohol by volume was greater than reported on the product or 2) the rate of evaporation was overestimated by manufacturers. As proposed in our research question, we wanted to consider the possibility that manufacturers underreport expiration dates simply due to a lack of empirical evidence and stringent guidelines. In that case, our first hypothesis would be supported, and it is possible that all samples had a greater initial concentration of ethyl alcohol than reported by manufacturers. 


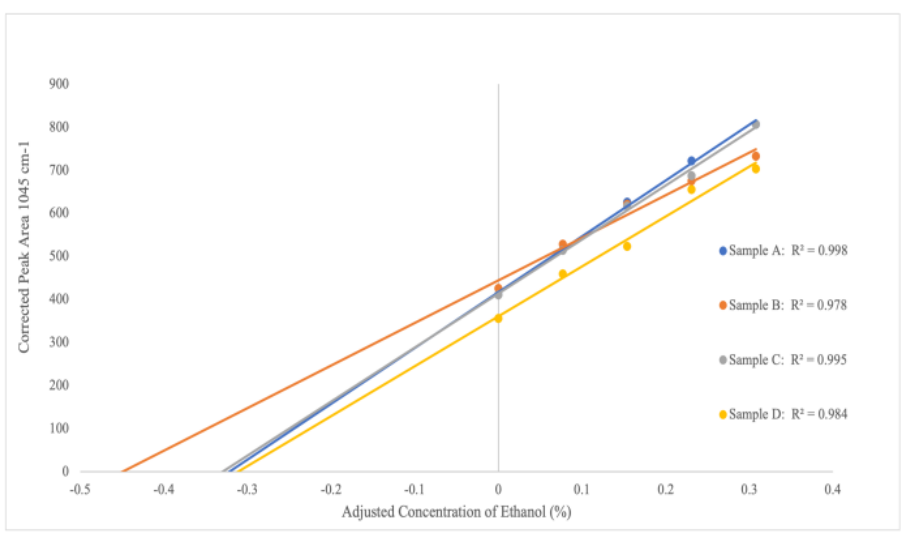

Figure 5: Standard addition calibration curves.

Table 2: The Adjusted Initial Ethyl Alcohol Volume (Ve, mL) and Ethyl Alcohol Concentration (v/v) in Samples A-D

\begin{tabular}{ccccc}
\hline Sample & $\begin{array}{c}{[\mathrm{X}]_{\mathrm{i}} \text { EtOH in }} \\
13 \mathrm{~mL} \text { sample }(\%)\end{array}$ & $\begin{array}{c}\mathrm{Calc.} \mathrm{EtOH} \\
(\% \mathrm{v} / \mathrm{v})\end{array}$ & $\begin{array}{c}\text { Reported EtOH } \\
(\% \mathrm{v} / \mathrm{v})\end{array}$ & $\begin{array}{c}\text { Total Loss } \\
(\% \mathrm{v} / \mathrm{v})\end{array}$ \\
\hline A & $0.321 \pm 0.015$ & $63.2 \pm 3.9$ & 68.00 & 4.78 \\
B & $0.330 \pm 0.020$ & $64.5 \pm 5.6$ & 70.00 & 5.49 \\
C & $0.312 \pm 0.040$ & $61.8 \pm 9.9$ & 62.00 & 0.23 \\
D & $0.449 \pm 0.057$ & $80.6 \pm 13.4$ & 75.00 & -5.60 \\
\hline
\end{tabular}

To calculate the uncertainty in the determined ethanol concentrations for each of the samples, the standard uncertainty of the $\mathrm{x}$-intercept was calculated using equation 2 :

$$
u_{x}=\frac{s_{y}}{|m|} \sqrt{\frac{1}{n}+\frac{\bar{y}^{2}}{m^{2} \sum\left(x_{i}-\bar{x}\right)^{2}}}
$$

where $\mathrm{s}_{\mathrm{y}}$ is the standard deviation of $\mathrm{y}$ (peak area), $\mathrm{m}$ is slope, and $\mathrm{n}$ is the number of points in the standard addition curve. This error was then propagated along with errors in the syringe in order to determine the absolute uncertainty. It is important to note that while the calculated percentages of ethanol for Samples B, C, and D fall within the acceptable range for efficacy, after accounting for error in the analysis, we are not certain about the effectiveness of these expired hand sanitizers.

There are several limitations to this study, most of all, the absence of preliminary empirical evidence. The expiration dates of Samples A-D were printed on the bottle; however, the date of manufacture was not readily available per the National Drug Code Directory (NDCD). ${ }^{31}$ One sample we proposed to study had an expiration date prior to 2015, however, the exact date and initial ethyl alcohol concentration were not readable on the packaging. We were able to decode the Lot Number but this product is no longer manufactured. The information we needed had been archived on NDCD or its expiry may exceed the electronic record information system. Another limitation of our study is the limited number of samples. We could not establish a systematic method for obtaining samples as they often came from friends and family and are not commercially available. While the concentration of ethanol can be quantitatively analyzed, the potential causes for the rate at which this evaporation occurred were unable to be studied. Each sample had a different brand, bottle, initial percentages of ethanol, and percentage of air in the bottle. These differences may have contributed to ethanol loss and further research should be done to analyze the brand specific ethanol loss over time to shed light on these variables. With a weaker seal on the bottle, more surface area exposed to air within the bottle, or storage at higher temperatures, ethanol may evaporate at faster rates causing a decline in efficacy. However, this exemplifies the significance of our study as we prepared this data with consideration for household use of these products rather than laboratory or institutional use. A final limitation of our study is that we based our signal intensity and predicted ethyl alcohol concentration based on the peak area of the primary alcohol C-O stretch (ROI) on FT-IR. Based on the ingredients list, it is possible that these peaks may have included other primary alcohols such as glycerol or isopropanol, although we minimized these concerns by performing a standard addition and considering ethyl alcohol as our primary ingredient by volume, we cannot definitively explain the effect of these ingredients.

Future research should take a longitudinal approach and measure the ethyl alcohol concentration over multiple years. Alternatively, future researchers may develop a procedure to "artificially age" samples of hand sanitizer. We recommend that future researchers attempt to trace back information on their samples by getting in touch with the manufacturer directly for Safety Data Sheets and data on the timing of production. If future studies can establish a firm manufacture date, they could create a predictive model with rate analysis for which hand sanitizer falls below the $60 \%(\mathrm{v} / \mathrm{v})$ threshold of ethyl alcohol concentration.

\section{CONCLUSIONS}

This study provides an accurate FT-IR determination of the ethanol percentage in hand sanitizer by using a standard addition curve to account for the matrix effect, not assessed in previous studies. ${ }^{26,27}$ While the expired samples analyzed in this study may still remain effective, the propagated error values overlap with the minimal $60 \%(\mathrm{v} / \mathrm{v})$ ethanol threshold and the efficacy is not certain. The ethanol concentration of Sample A also suggests that manufacturers may use a higher percentage of alcohol when creating the hand sanitizer in order to account for this loss over time and ensure efficacy. Further analyses should be conducted to determine the concentration of glycerol in expired hand sanitizer as it may play a more important role in determination of efficacy. As the boiling points of ethanol and water are $78.2{ }^{\circ} \mathrm{C}$ and $100{ }^{\circ} \mathrm{C}$ respectively, they will evaporate at a faster rate than glycerol with a boiling point $290{ }^{\circ} \mathrm{C} .{ }^{32}$ This will cause the concentration of glycerol to increase in the 
samples and possibly cause interference with ethanol concentration analysis using FT-IR.

This study has brought light to hand sanitizer expiration dates of which no study, to our knowledge, has been published. It is important to bring these findings into the public view as hand hygiene is a crucial aspect of preventing bacterial and viral diseases such as COVID-19. Members of all communities need to be educated and pay closer attention to these expiration dates as a false sense of security can be dangerous, especially during a pandemic. We hope that further research is conducted to investigate the effects of bottle seals, storage temperature, and other important facets that play a role in the continuation of the shelf life of hand sanitizer.

\section{AUTHOR INFORMATION}

\section{*Corresponding Author:}

Ling Hao, PhD - Assistant Professor, Department of Chemistry, The George Washington University, Science and Engineering Hall, 800 22nd Street NW, Washington, D.C. 20052, USA orcid.org/0000-0002-0106-5266.

Email: linghao@gwu.edu

\section{Author contributions:}

$\uparrow$ These authors contributed equally to this work

\section{Notes}

The authors declare no competing financial interest.

\section{ACKNOWLEDGMENTS}

This project was supported by the Department of Chemistry at the George Washington University, through Chem 2123W course: Introductory Quantitative Analysis Laboratory. We would also like to thank the Department of Chemistry at The George Washington University for supplying the facilities and instrumentation used in this study. Our Chem $2123 \mathrm{~W}$ course in 2021 Spring was featured on the GW News:

(https://gwtoday.gwu.edu/kitchen-chemistry-home-

experiments-enhance-remote-learning)

\section{REFERENCES}

1. Kratzel, A. et al. Inactivation of Severe Acute Respiratory Syndrome Coronavirus 2 by WHORecommended Hand Rub Formulations and Alcohols. Emerg. Infect. Dis. 26, 1592-1595 (2020).

2. CDC. Core Infection Prevention and Control Practices for Safe Healthcare Delivery in All Settings Recommendations of the Healthcare Infection Control Practices Advisory Committee. Cdc 1-15 (2017).
3. Yan, Y. et al. Consensus of Chinese experts on protection of skin and mucous membrane barrier for health-care workers fighting against coronavirus disease 2019. Dermatol. Ther. 33, 1-7 (2020).

4. Berardi, A. et al. Hand sanitisers amid CoViD-19: A critical review of alcohol-based products on the market and formulation approaches to respond to increasing demand. Int. J. Pharm. 584, (2020).

5. Chakraborti, R. \& Roberts, G. Anti-Price Gouging Laws, shortages, and COVID-19: Big Data Insights from Consumer Searches. Soc. Sci. Humanit. Open (2020).

6. Tiwari, A. Mystery Date : Advocating for a Harmonized System of Expiration Date Labeling of Food. Vanderbilt J. Transnatl. Law 49, 1447-1475 (2016).

7. Secondi, L. Expiry dates, consumer behavior, and food waste: How would Italian consumers react if there were no longer 'best before' labels? Sustain. 11, 1-15 (2019).

8. U.S. Food \& Drug Administration. Q \& A for Consumers | Hand Sanitizers and COVID-19. 12/15/2020.

9. Morton, H. E. the Relationship of Concentration and Germicidal Efficiency of Ethyl Alcohol. Ann. N. Y. Acad. Sci. 53, 191-196 (1950).

10. Jain, V. M., Karibasappa, G. N., Dodamani, A. S., Prashanth, V. K. \& Mali, G. V. Comparative assessment of antimicrobial efficacy of different hand sanitizers: An in vitro study. Dent. Res. J. (Isfahan). 13, 424-431 (2016).

11. Ingram, L. O. Ethanol tolerance in bacteria. Crit. Rev. Biotechnol. 9, 305-319 (1989).

12. Rigomier, D., Bohin, J. P. \& Lubochinsky, B. Effects of ethanol and methanol on lipid metabolism in Bacillus subtilis. J. Gen. Microbiol. 121, 139-149 (1980).

13. Golin, A. P., Bhsc, D. C. \& Ghahary, A. Hand sanitizers: A review of ingredients, mechanisms of action, modes of delivery, and efficacy against coronaviruses. (2020).

14. Kampf, G. \& Kramer, A. Epidemiologic background of hand hygiene and evaluation of the most important agents for scrubs and rubs. Clin. Microbiol. Rev. 17, 863-893 (2004).

15. Fendler, E. J. et al. The impact of alcohol hand sanitizer use on infection rates in an extended care facility. Am. J. Infect. Control 30, 226-233 (2002).

16. PubChem Compound Summary for CID 702, Ethanol. Natl. Cent. Biotechnol. Inf. (2021).

17. PubChem Compound Summary for CID 962, Water. Natl. Cent. Biotechnol. Inf. (2021).

18. WHO. WHO Guidelines on Hand Hygiene in Health Care.

19. Sattar, S. A., Ali, M. \& Tetro, J. A. In Vivo comparison of two human norovirus surrogates for testing ethanolbased handrubs: The mouse chasing the cat! PLoS One 6, 1-6 (2011).

20. Nogueira, S. A. et al. Redox titration on foldable paperbased analytical devices for the visual determination of 
alcohol content in whiskey samples. Talanta 194, 363369 (2019).

21. Lobo, M. J., Miranda, A. J. \& Tuñón, P. Flow-Injection Analysis of Ethanol with an Alcohol DehydrogenaseModified Carbon Past Electrode. Electroanalysis 8, 932-937 (1996).

22. Shkotova, L. V., Soldatkin, A. P., Gonchar, M. V., Schuhmann, W. \& Dzyadevych, S. V. Amperometric biosensor for ethanol detection based on alcohol oxidase immobilised within electrochemically deposited Resydrol film. Mater. Sci. Eng. C 26, 411-414 (2006).

23. Cordell, R. L., Pandya, H., Hubbard, M., Turner, M. A. \& Monks, P. S. GC-MS analysis of ethanol and other volatile compounds in micro-volume blood samplesquantifying neonatal exposure. Anal. Bioanal. Chem. 405, 4139-4147 (2013).

24. Boyaci, I. H., Genis, H. E., Guven, B., Tamer, U. \& Alper, N. A novel method for quantification of ethanol and methanol in distilled alcoholic beverages using Raman spectroscopy. J. Raman Spectrosc. 43, 11711176 (2012).

25. NIST. NIST Standard Reference Data Program: Ethanol. 2018 (2018).

26. Evans, Kieran. Bohman, A. Quantification of ethanol and isopropanol in alcohol-based hand sanitizers. PerkinElmer, Inc. 1-3 (2020).

27. Shimadzu. Application news. Biometric Technol. Today 15, 3 (2007).

28. Jordan, M. A., Choksi, D., Lombard, K. \& Patton, L. R. Development of Guidelines for Accurate Measurement of Small Volume Parenteral Products Using Syringes. Hosp. Pharm. (2019) doi:10.1177/0018578719873869.

29. Infrared Spectroscopy: Demonstrating the accuracy of transmittance measurements for high refractive index materials. PerkinElmer, Inc. https://www.perkinelmer.com/labsolutions/resources/docs/TCH_FrontierOptica.pdf (2010).

30. Harris, D. C. Quantiative Chemical Analysis. (W. H. Freeman and Company, 2010).

31. U.S. Food \& Drug Administration. National drug code directory.

32. PubChem Compound Summary for CID 753, Glycerol. Natl. Cent. Biotechnol. Inf. (2021). 